\title{
Teleradiological implementation as a decision support system at an accident service unit in a tertiary care hospital in Sri Lanka
}

\author{
D.H. Jayakody ${ }^{1}{ }^{*}$, R.B. Marasinghe ${ }^{2}$ \\ 1 Postgraduate Institute of Medicine, University of Colombo, Sri Lanka \\ 2 Faculty of Medical Sciences, University of Sri Jayewardenepura, Sri Lanka \\ *harsha_jayakody@yahoo.com \\ https://orcid.org/0000-0001-9151-3968
}

\begin{abstract}
Introduction: Recent developments in teleradiology have introduced various technologies including PACS (Picture Archiving and Communication System), DICOM (Digital Imaging and Communication in Medicine), \& Oviyam, helping healthcare institutions to make a smooth transition to a digital radiology infrastructure, which is more efficient and effective compared to traditional radiology systems. However, given the potentially unique socio-political aspects, perspectives, \& infrastructure, feasibility of a digital radiology system and the adaptable technologies can vary among different types of healthcare institutions in different regions around the world. This study attempted to make an optimal teleradiological implementation at the Accident Service Unit of Colombo South Teaching Hospital, a government tertiary care hospital in Sri Lanka.
\end{abstract}

Methods: The information and subjective opinions gathered from different channels in two phases, in the form of a questionnaire, in order to make an assessment on the feasibility of transition to a digital teleradiology system. After a comprehensive analysis of requirements and constraints, a teleradiological system was implemented at the hospital. A post-implementation assessment was carried out using a questionnaire in the second phase of the study, which was conducted among all the medical professionals using the system.

Results: Based on the post implementation assessment, more than $50 \%$ of the participants indicated that the ability to view radiology images at a distance with an acceptable interpretation quality, improves patient care and management of clinical data. More than $66.7 \%$ of participants indicated that the newly implemented teleradiology system improved the productivity and quality of patient care and enhanced the ability to coordinate and proceed with patient care.

Conclusions: According to the post-implementation evaluation, the teleradiological solution implemented in this study has enabled a better, effective, and efficient delivery of radiology services at the tertiary care setting.

Keywords: Teleradiology, PACS, DICOM 